\title{
APPENDIX 2 :
}

\section{THE BALATONFÜRED ALPHABET OF COSMOLOGY}

Firstly, Aaronson let us recall,

For his death was a blow to us all,

But his papers, J. Mould,

His colleague of old,

Will present in due time, in the Hall.

With B, let us contemplate Bubbles, Which have brought to our theory some troubles ;

Distance now must be counted,

So turn we, undaunted,

To red shift, and that constant of Hubble's.

Contrariwise, we've $\mathrm{C}$ for Cluster,

Where galaxies closely do muster,

Both richly and poorly,

Observing them, surely,

Will bring the keen scholar great lustre.

In the microwave, Dipoles we see, Which we plot, $\Delta \mathrm{T}$ upon $\mathrm{T}$;

Then, to keep the score level,

Kofman draws us the Devil,

And a haloed, hirsute Deity.

With E, Einstein comes into sight, Whose theories once brought delight,

Now we think, with respect,

He was not quite correct ;

But who, out of hundreds, is right ?

With F, we pursue the Fifth Force,

Of many a question the source ;

Profound explanations

Of its implications

Fujii will report in due course.

$\mathrm{G}$ for Galaxies, spiral, elliptic, 
Or lens-shaped, of origin cryptic ;

And what is this factor

Called the Great Attractor,

Sited southerly from the ecliptic ?

$\mathrm{H}$, of course, our Hungarian Hosts ;

To Sandor and György drink we toasts,

And to the SZOT hotel some !

They made us so welcome,

Down here on Lake Balaton's coasts,

Inflation and the Infrared

Are topics where much may be said,

The data from IRAS

Are sure to inspire us,

I will argue no more on that head !

$\mathrm{J}$ are our two Journalists

One Nature's, one New Scientist's

Reporting our labours

To colleagues and neighbours,

To tell them what fun they have missed !

$K$ is the letter we choose

To denote certain constants we use,

(Since I've no other place,

I will borrow this space,

To honour Urania - our muse !)

L for lenses (of type gravitational),

Which, occuring in fields observational,

May prove a delusion

And lead to confusion

And provoke us to anger irrational

Here at $\mathrm{M}$ let controversists chatter,

Looking far where the galaxies scatter :

"In this vast universe

Is a substance perverse :

Is it cold ? Is it Dark ? Does it Matter ?"

N-bodied is Frenk's simulation

Presentig dark halo formation,

But he gave it so fast

We were quite lost at last,

Though we noted his good correlation ! 
O, our Outing - not just a diversion, But to Eötvös's work an excursion ; In the Tihany museum, For people to see 'em,

Are his balances, based upon torsion.

$\mathbf{P}$ for Posters, where research assorted

In format of Print is reported, (And addenda in ink

Reveal a rethink

Of a theory in mid-stream aborted).

Next Quasars, phenomena curious ;

Doubtful data could here prove injurious, So first we must know

(As the Cambridge team show)

How to weed from our survey the spurious!

Relativity now has to follow,

Whose details may prove hard to swallow,

But work through the night,

And maybe we might

Understand somewhat better tomorrow !

With S, we have reached cosmic Strings,

Some are Superconducting - strange things-

Some are infinite, straight,

And some, I can state,

Are loops (topologically, rings).

$\mathrm{T}$ is, of course, Telescope ;

From the earth we can now hardly cope :

But a space device can,

So, according to plan,

One will fly in the " 90 "s (we hope !)

Filled with Unknowns is our Universe, To the scholar both blessing and curse, For each theory one tenders

New questions engenders,

-And Uncertainty grows ever worse !

V next : Very Large the Arrays

That on radio frequencies gaze ;

Though each point - or pixel -

Will not form a thick cell, 
The picture builds up over days.

$\mathrm{W}$ - and arrived at this junction,

The brain shows a marked lack of gumption :

But to counter a void,

What else should be deployed

But its complement, viz : the Wall function ?

And now X-ray background (alas !)

Does it emanate from dispersed gas

Abundant in heat?

Or from sources discrete

Of baryons, heavy in mass?

[wh]Y is the questioning particle, A most indispensible article !

For, did they not ask,

The researchers' task

Would be, frankly speaking, unstartable !

$\mathrm{Z}$ - the end at last ! So, ere I go (which

I must), I will just let you know which

Conference team is the best :

Come, proclaim them with zest :

Fifteen names from Audouze to Zel'dovich !

Vera Rich

Nature

London, UK 